Research Article

\title{
Effect of gliclazide on cardiovascular risk factors involved in split-dose streptozotocin induced neonatal rat model: a chronic study
}

\author{
I. Mohammed Salman*, Md. Naseeruddin Inamdar
}

\begin{abstract}
a Department of Pharmacology, Al-Ameen College of Pharmacy, Bangalore - 560027, India
\end{abstract}

Received: 8 October 2012 Accepted: 23 October 2012

*Correspondence to:

I. Mohammed Salman, Email: mdsalman1985@gmail.com

\begin{abstract}
Background: The present study aimed at evaluating the effect of gliclazide on cardiovascular risk factors involved in type 2 diabetes mellitus using $n-S T Z$ rat model on a long term basis.

Methods: The diabetic model was developed using a split dose of streptozotocin $(50 \mathrm{mg} / \mathrm{kg})$ intraperitoneally on $2^{\text {nd }}$ and $3^{\text {rd }}$ postnatal days. The diabetic rats were treated orally with gliclazide suspension at the dose of 10 $\mathrm{mg} / \mathrm{kg}$ for 90 days. Cardiovascular risk factors such as systolic blood pressure, heart rate, lipid profile, creatine kinase and lactate dehydrogenase were evaluated at regular intervals along with fasting blood glucose (FBG) and oral glucose tolerance test.
\end{abstract}

Results: Gliclazide did not alter FBG however improved the impaired glucose tolerance. The gliclazide treated rats did not develop hypertension and there was a significant difference $(p<0.001)$ at the end of treatment when compared to the diabetic group which could be due to free radical scavenging property of gliclazide. Gliclazide treatment in n-STZ model was found to be effective in preventing hypertension, creatine kinase and lactate dehydrogenase activity. Also gliclazide was found to have beneficial effects on the impaired glucose tolerance, dyslipidaemia, adiposity index and total fat pad weight.

Conclusions: To improve and prevent the cardiovascular risk factors involved in Type II diabetic patients, gliclazide could be clinically beneficial.

Keywords: n-STZ rat model, streptozotocin, gliclazide, diabetes, hypertension, cardiovascular risk factors

\section{INTRODUCTION}

Type 2 diabetes mellitus (T2DM) is a complex, heterogeneous, polygenic disease characterized mainly by insulin resistance and pancreatic $\beta$ - cell dysfunction. ${ }^{1}$ Appropriate experimental models are essential for understanding the underlying mechanisms of diabetes and its complications. Investigations have unsuccessfully tried to recreate in experimental animals the cardiovascular complications of diabetes seen in humans. Sulfonylureas have remained the mainstay of antidiabetic therapy for almost three decades. ${ }^{1}$ However the benefits of sulfonylureas beyond glycemic control has to be investigated. Gliclazide a second-generation sulfonylurea was preferred in the present study due to its vascular benefits beyond glycemic control. ${ }^{2}$ Gliclazide possesses free radical scavenging property due to presence of aminoazabicyclo-octane ring, which is absent in other sulfonylureas. $^{2}$
In T2DM patients elicited a greater suppression of hepatic glucose production. ${ }^{3}$ Gliclazide, but not glibenclamide, glimepiride, glipizide or tolbutamide, inhibited low density lipoprotein oxidation and enhanced total plasma antioxidant capacity. Thus, gliclazide possesses antioxidant properties that produce measurable clinical effects at therapeutic doses. ${ }^{4}$ Gliclazide reduces DNA damage and improves DNA repair in diabetic patients, thus it may protect against the oxidative stressrelated chronic diabetes complications, including cancer, by decreasing the level of DNA damage induced by reactive oxygen species. ${ }^{5}$ Gliclazide reduces oxidative stress $^{6}$ in enhanced nitric oxide (NO)-mediated vasodilatation. Also decrease in blood pressure was shown after gliclazide treatment which was mediated through NO- mediated vasodilatation. ${ }^{7}$ Improving the oxidative status of patients by using gliclazide for its hemovascular properties independent of its hypoglycemic action has led to improvements in oxidative stress, 
hypercoagulability, endothelial function and platelet reactivity which have not been demonstrated by other sulphonylureas. In T2DM, where hyperglycemia and oxidation are fundamental to the ultimate thrombotic complications of diabetes, agents such as gliclazide with antioxidant activities may have an enhanced therapeutic role. $^{2}$ Among the T2DM rodent models used n-STZ model (neonatal streptozotocin) was found to be more appropriate which resembles human T2DM more than other models available. ${ }^{8}$ Hence in this study, the long term effect of gliclazide on cardiovascular complications involved in T2DM was studied using n-STZ rat model.

\section{METHODS}

Streptozotocin (STZ) was purchased from Sigma Aldrich, Germany. Biochemical kits were procured from Swemed Diagnostics, Bangalore India. Gliclazide (Torrent Pharmaceuticals, India) suspension was prepared using $2 \%$ Tween $80^{\circledR}$ in water. All the reagents and chemicals used in this study were of analytical grade.

Male Wistar neonatal rats were procured from the in house animal facility. The animals were housed in standard polypropylene cages (three rats/cage) and maintained under controlled room temperature $\left(22 \pm 2{ }^{\circ} \mathrm{C}\right)$ and humidity $(55 \pm 5 \%)$ with $12: 12 \mathrm{~h}$ light and dark cycle. All the rats were provided with commercially available rat normal pellet diet (Amrut Diet, New Delhi) and water ad libitum. The studies were performed with the approval of Institutional Animal Ethics Committee (IAEC) of AlAmeen College of Pharmacy, Bangalore, India (Reference No. AACP/IAEC/M-94/2007).

\section{Experimental design}

STZ was administered to neonatal rats $(n=24)$ on the $2^{\text {nd }}$ and $3^{\text {rd }}$ postnatal day in the dose of $50 \mathrm{mg} / \mathrm{kg} /$ day intraperitoneally ${ }^{9}$, and vehicle (citrate buffer) was administered to control group $(n=9)$. After 28 days, the rats pups were weaned and fasting blood glucose (FBG) and oral glucose tolerance test (OGTT), in response to 2 $\mathrm{g} / \mathrm{kg}$ oral glucose using $50 \%$ (w/v) glucose solution, were carried out at 8 and 12 weeks. The animals having FBG $\geq$ $100 \mathrm{mg} / \mathrm{dl}$ along with impaired glucose tolerance at 12 weeks were considered to be diabetic and included in the study. Twelve weeks post n-STZ administration; the diabetic rats were divided into two groups. A group of rats $(n=12)$ was administered $2 \%$ Tween $80^{\circledR}$ in water (diabetic control) while the other group of rats $(n=12)$ was treated orally with gliclazide $(10 \mathrm{mg} / \mathrm{kg})$ suspension using $2 \%$ Tween $80^{\circledR}$ in water solution, for about 90 days. The first day of gliclazide treatment was considered as day 0 of the study.

The biochemical parameters were estimated using standard kits. The blood pressure (BP) and heart rate (HR) was determined using tail cuff method by averaging 5-8 individual readings (Power lab data acquisition system, Australia). The body weight of the animals was recorded throughout the study period.

\section{Collection of blood and biochemical analysis}

Blood samples were collected from retro-orbital plexus of the rats under light ether anesthesia using capillary tubes into eppendorf tubes and were left aside to clot. The blood sample was centrifuged (5min, $8000 \mathrm{rpm}$ ) and the serum was analyzed for glucose (GOD-POD), triglycerides (GPO-POD) total cholesterol and HDL cholesterol (CHOD-POD) levels by using commercially available colorimetric diagnostic kits using Artos semiauto analyzer (Swemed diagnostics, Bangalore, India). For OGTT estimation, blood samples were collected at 0 , $15,30,60,90$ and 120 minutes.

\section{Estimation of enzyme activity}

Creatine Kinase-MB (CK-MB) and Lactate dehydrogenase (LDH) estimations were carried out by diluting the serum samples using potassium phosphate buffer $\mathrm{pH} 7.4 \quad(100 \mathrm{mmol} / \mathrm{L})$ using commercially available kits (Swemed diagnostics, Bangalore India).

\section{Blood pressure and Heart rate}

The rats were taken to the experimental room 4 hours before systolic blood pressure (SBP) recording. The rats were kept in a restrainer; tail cuff along with pulse detector connected to a data acquisition system (Power lab, Australia) was placed at the base of the tail. After confinement in a holder for 10-15 minutes at room temperature, tail pulsations were large enough for accurate estimation of SBP. Each recording was obtained by averaging 5-8 individual readings. The heart rate (HR) was calculated simultaneously.

\section{Adiposity index}

The body weight of the animals was recorded every week throughout the study period. The total weight gain in the study period was calculated using the difference between the final body weight and the initial body weight observed.

At the end of the study, perirenal, mesenteric, epididymal and pericardial fat pads were isolated from all the rats. The fat pads were weighed immediately to obtain wet FPW and are expressed in $\mathrm{g}$ per $100 \mathrm{~g}$ of body weight. The sum of all FPW was taken as total fat pad weight (TFPW). TFPW value represents the overall adipose mass present in the rats.

The adiposity index (ADI) was calculated using the following formula ${ }^{7}$ :

$$
\mathrm{ADI}=\frac{\text { TFPW }}{(\text { body weight }- \text { TFPW) }} \times 100
$$




\section{Statistical analysis}

The results are expressed as mean \pm S.E.M. One-way ANOVA followed by multiple comparison test (TukeyKramer multiple comparison tests) was applied between groups. A value of $p<0.05$ was considered statistically significant.

\section{RESULTS}

The treatment with gliclazide was initiated at $12^{\text {th }}$ week after n-STZ administration, when the FBG levels were elevated slightly and glucose tolerance was impaired. The impaired glucose tolerance (IGT) was found to have improved during OGTT indicated by area under the curve for glucose (AUCg) when compared to the diabetic group following glicalzide treatment for 90 days; however it did not decrease FBG (Table 1).

Total cholesterol (TC) and atherosclerotic index (AI) levels significantly rose in the diabetic group only in the later part of the study when compared with the control group. Gliclazide lowered the AI significantly $(p<0.01)$ after 90 days of treatment (Table 1 ). While triglycerides (TG) increased significantly in diabetic group on Days 0, 30 and 90; glicalzide seems to have no effect on TG.

Table 1: Effect of gliclazide on glucose and lipid profile in n-STZ diabetic rat model.

\begin{tabular}{|c|c|c|c|c|c|c|}
\hline $\begin{array}{c}\text { Treatment } \\
\text { Days }\end{array}$ & Groups & $\begin{array}{c}\text { FBG } \\
(\mathrm{mg} / \mathrm{dL})\end{array}$ & $\begin{array}{c}\mathrm{AUCg} \\
(\mathrm{dL} \times \mathrm{min} / \mathrm{g})\end{array}$ & $\begin{array}{c}\text { TG } \\
(\mathrm{mg} / \mathrm{dL})\end{array}$ & $\begin{array}{c}\text { TC } \\
(\mathrm{mg} / \mathrm{dL})\end{array}$ & AI \\
\hline \multirow{3}{*}{0} & Control & $66.28 \pm 1.96$ & $13.9 \pm 0.12$ & $84.24 \pm 5.98$ & $65.3 \pm 4.46$ & $0.63 \pm 0.11$ \\
\hline & Diabetic & $123.55 \pm 5.28^{c}$ & $29.86 \pm 1.46^{c}$ & $116.7 \pm 6.78^{c}$ & $75.97 \pm 1.44$ & $0.61 \pm 0.04$ \\
\hline & Gliclazide & $129.15 \pm 10.83$ & $30.46 \pm 1.25$ & $128.2 \pm 2.15$ & $71.42 \pm 1.45$ & $0.61 \pm 0.05$ \\
\hline \multirow{3}{*}{30} & Control & $70.98 \pm 1.08$ & $14 \pm 0.1$ & $83.58 \pm 5.09$ & $67.79 \pm 2.6$ & $0.47 \pm 0.04$ \\
\hline & Diabetic & $125.62 \pm 6.60^{c}$ & $31 \pm 2.43^{c}$ & $117.1 \pm 4.77^{b}$ & $85.5 \pm 5.32^{a}$ & $0.71 \pm 0.07$ \\
\hline & Gliclazide & $126.78 \pm 5.74$ & $27.66 \pm 1.52$ & $122.2 \pm 9.08$ & $92.33 \pm 3.82$ & $0.91 \pm 0.1$ \\
\hline \multirow{3}{*}{90} & Control & $73.67 \pm 1.10$ & $15.07 \pm 0.15$ & $81.68 \pm 3.82$ & $74.75 \pm 1.00$ & $0.62 \pm 0.02$ \\
\hline & Diabetic & $130.79 \pm 3.28^{c}$ & $33.36 \pm 1.16^{c}$ & $117 \pm 4.06^{c}$ & $93.19 \pm 3.12^{b}$ & $1.23 \pm 0.08^{c}$ \\
\hline & Gliclazide & $124.15 \pm 3.29$ & $28.12 \pm 1.27^{* *}$ & $118 \pm 5.14$ & $94.02 \pm 3.62$ & $0.89 \pm 0.08^{* *}$ \\
\hline
\end{tabular}

Values are Mean \pm SEM; $n=9$ for control group and $n=12$ for diabetic and gliclazide group

The abbreviations denote FBG: Fasting blood glucose, AUCg: Area under the curve for glucose values from 0-120 minutes, TG: Triglycerides, TC: Total cholesterol, AI: Atherogenic index (AI = TC-HDL cholesterol divided by HDLcholesterol). Diabetic group Vs Control group shown as a $(p<0.05), \mathrm{b}(p<0.01), \mathrm{c}(p<0.001)$. Gliclazide treated group Vs Diabetic group shown as $*(p<0.05), * *(p<0.01), * * *(p<0.001)$.

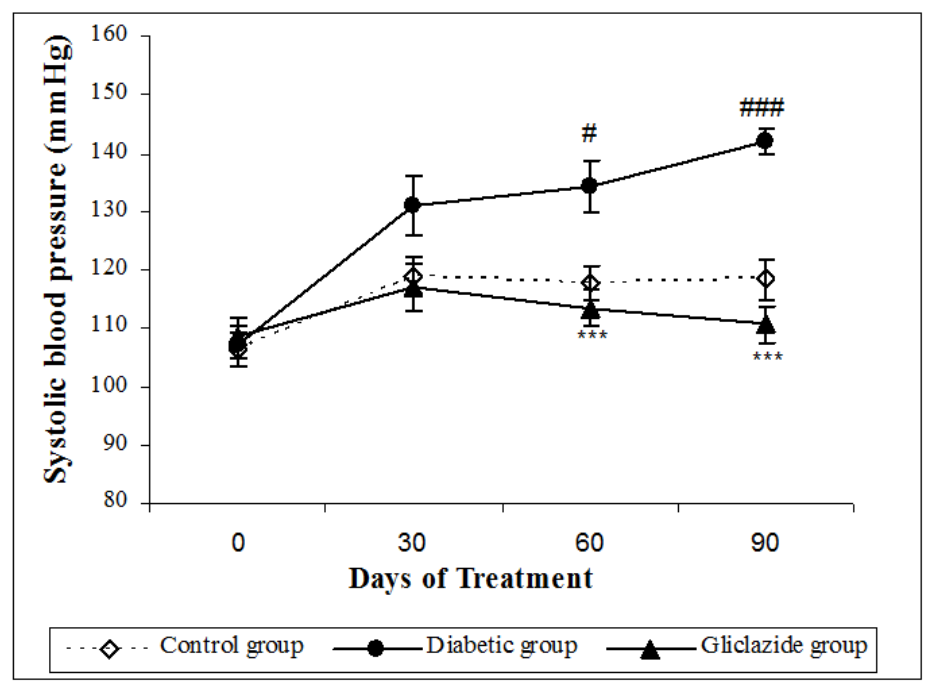

Figure 1: Effect of gliclazide on blood pressure in n-STZ diabetic rat model.

Values are expressed as Mean \pm SEM; $n=9$ for control group and $n=12$ for diabetic and gliclazide group

Diabetic group Vs Control group shown as \# $(p<0.05)$, \#\#\# $(p<0.001)$.

Gliclazide treated group Vs Diabetic group shown as $* * *(p<0.001)$. 
Table 2: Effect of gliclazide on CK-MB and LDH levels in n-STZ diabetic rat model.

\begin{tabular}{|c|c|c|c|}
\hline $\begin{array}{c}\text { Treatment } \\
\text { Days }\end{array}$ & Groups & $\begin{array}{c}\text { CK-MB } \\
\text { activity U/L }\end{array}$ & $\begin{array}{c}\text { LDH } \\
\text { activity U/L }\end{array}$ \\
\hline \multirow{3}{*}{0} & Control & $1292.5 \pm 74.1$ & $829.01 \pm 91.63$ \\
\cline { 2 - 4 } & Diabetic & $1822.7 \pm 64.4^{\mathrm{c}}$ & $1284.5 \pm 84.33^{\mathrm{a}}$ \\
\cline { 2 - 4 } & Gliclazide & $1856.6 \pm 94.2$ & $1203.8 \pm 111.08$ \\
\hline \multirow{3}{*}{30} & Control & $1406.9 \pm 84.9$ & $808.97 \pm 33.81$ \\
\hline \multirow{2}{*}{90} & Diabetic & $2204.1 \pm 97.4^{\mathrm{c}}$ & $2209.6 \pm 170.8^{\mathrm{c}}$ \\
\cline { 2 - 4 } & Gliclazide & $1760.8 \pm 159.7^{*}$ & $1061.7 \pm 79.6^{* * * *}$ \\
\hline \multirow{2}{*}{90} & Control & $1528.3 \pm 41.3$ & $949.26 \pm 50.20$ \\
\cline { 2 - 4 } & Diabetic & $2092 \pm 73^{\mathrm{c}}$ & $2134.4 \pm 153.3^{\mathrm{c}}$ \\
\hline & Gliclazide & $1012.4 \pm 73.8^{* * *}$ & $1524.7 \pm 116.78^{* *}$ \\
\hline
\end{tabular}

Values are Mean \pm SEM; $n=9$ for control group and $n=12$ for diabetic and gliclazide group

The abbreviations denote CK-MB: Creatine kinase; LDH: lactate dehydrogenase.

Diabetic group Vs Control group shown as a $(p<0.05), \mathrm{b}(p<0.01), \mathrm{c}(p<0.001)$.

Gliclazide treated group Vs Diabetic group shown as * $(p<0.05), * *(p<0.01), * * *(p<0.001)$

Table 3: Effect of gliclazide on organ weight in n-STZ diabetic rat model.

\begin{tabular}{|c|c|c|c|c|c|}
\hline \multirow{2}{*}{ Groups } & \multicolumn{4}{|c|}{ Organ weight (gms) /100 gm body weight } & \multirow{2}{*}{$\begin{array}{c}\text { Heart: Body weight } \\
\text { ratio }\end{array}$} \\
\cline { 2 - 6 } & Kidney & Heart & Liver & Spleen & $0.32 \pm 0.01$ \\
\hline Control & $0.68 \pm 0.01$ & $0.30 \pm 0.00$ & $3.75 \pm 0.08$ & $0.30 \pm 0.0001$ \\
\hline Diabetic & $0.72 \pm 0.03$ & $0.38 \pm 0.03^{\mathrm{a}}$ & $3.04 \pm 0.20^{\mathrm{b}}$ & $0.30 \pm 0.01$ & $0.0038 \pm 0.00004^{\mathrm{a}}$ \\
\hline Gliclazide & $0.66 \pm 0.02$ & $0.32 \pm 0.01$ & $3.57 \pm 0.15^{*}$ & $0.28 \pm 0.01$ & $0.0032 \pm 0.0001$ \\
\hline
\end{tabular}

Values are expressed as Mean \pm SEM; $n=9$ for control group and $n=12$ for diabetic and gliclazide group; Diabetic group Vs Control group shown as a $(p<0.05), \mathrm{b}(p<0.01)$; Gliclazide treated group Vs Diabetic group shown as $*(p<0.05)$.

Table 4: Effect of gliclazide on adiposity index in n-STZ diabetic rat model.

\begin{tabular}{|c|c|c|c|c|c|c|}
\hline \multirow{2}{*}{ Groups } & \multicolumn{4}{|c|}{ Fat Pad Weights (gms) /100 gm Body Weight } & Adiposity \\
\cline { 2 - 7 } & Perirenal & Mesenteric & Epididymal & Pericardial & $\begin{array}{c}\text { Total fat pad } \\
\text { weight }\end{array}$ & index (ADI) \\
\hline Control & $0.56 \pm 0.01$ & $0.77 \pm 0.02$ & $1.01 \pm 0.04$ & $0.07 \pm 0$ & $2.42 \pm 0.03$ & $2.46 \pm 0.03$ \\
\hline Diabetic & $0.46 \pm 0.05$ & $0.63 \pm 0.03$ & $0.90 \pm 0.06$ & $0.02 \pm 0^{\mathrm{c}}$ & $2.05 \pm 0.12^{\mathrm{a}}$ & $2.09 \pm 0.13^{\mathrm{a}}$ \\
\hline Gliclazide & $0.82 \pm 0.04^{* * *}$ & $0.71 \pm 0.05$ & $1.01 \pm 0.05$ & $0.04 \pm 0^{* * *}$ & $2.59 \pm 0.13^{* * *}$ & $2.66 \pm 0.14^{* * * *}$ \\
\hline
\end{tabular}

Values are expressed as Mean $\pm \mathrm{SEM} ; n=9$ for control group and $n=12$ for diabetic and gliclazide group Diabetic group Vs Control group shown as a $(p<0.05)$, $\mathrm{c}(p<0.001)$.

Gliclazide treated group Vs Diabetic group shown as $* * *(p<0.001)$.

The SBP increased significantly during the study in the diabetic group when compared to the control group. The gliclazide treatment attenuated the raise in SBP significantly when compared to the diabetic group (Figure 1). There was no significant change observed in the heart rate among the groups (Control, diabetic and gliclazide).

There was a significant rise in creatinine kinase (CK-MB) and lactate dehydrogenase (LDH) levels throughout the period in diabetic group and these levels were found to be decreased following gliclazide treatment (Table 2).

Organ weights, recorded at the end of treatment period, show that liver weight was increased in gliclazide treated rats compared to the diabetic group (Table 3). During the study period gliclazide treated rats show a significant increase $(p<0.01)$ in the body weight in comparison to the diabetic group. 
The improvement in IGT after gliclazide treatment can be correlated with the increase in total fat pad weight (TFPW) and adiposity index (ADI) (Table 4).

\section{DISCUSSION}

The observations in our study support the earlier reports that the gliclazide treatment did not significantly modify the plasma glucose levels in n0-STZ model where insulin was found to be deficient. ${ }^{10}$ Interestingly, elevated glucose levels were brought back to normal in n5-STZ model where insulin resistance was observed. ${ }^{10}$

Glycogen synthesis in muscle accounts for the major part of non-oxidative glucose metabolism during insulin stimulation. Gliclazide treatment in T2DM patients was found to be associated with a potentiation of both insulinmediated glucose disposal and insulin-stimulated glycogen synthase activity in skeletal muscle. ${ }^{11}$ Thus, the improvement in the IGT observed in the present study may be due to extrapancreatic effect of gliclazide as reported earlier. ${ }^{11,12}$

The improved IGT after chronic treatment in the present study was in line with reported effects of gliclazide in GK rats $^{13}$, where increase in beta cell insulin stores and increase in glucose induced insulin release was demonstrated in vivo which fit with the clinical data concluding that chronic gliclazide therapy augments the stimulated first phase insulin secretion in patients with T2DM by increasing islet sensitivity to glucose. ${ }^{14}$ However, the chronic treatment in the present study did not reduce the elevated FBG level in split dose n-STZ model.

Use of $100 \mathrm{mg} / \mathrm{kg}$ of STZ in split dose in the present study was still a higher dose when compared to the earlier study $^{15}$ reporting effectiveness of gliclazide in mild T2DM neonatal rat model using $60 \mathrm{mg} / \mathrm{kg}$ on $3^{\text {rd }}$ postnatal day in a single shot administration. Since the dose of gliclazide used in many studies ${ }^{10,11,15,16}$ and in the present study was $10 \mathrm{mg} / \mathrm{kg}$, the ineffectiveness of gliclazide may be due the reason that the dose of n-STZ used in this study may still be higher for checking effectiveness of sulfonylureas. Gliclazide was expected to reduce the elevated FBG levels in $n-S T Z$ model as glibenclamide was found to be effective in earlier study ${ }^{17}$ in the same model. Thus, the effectiveness of the sulfonylureas in the n-STZ model in bringing back elevated FBG level still remains unclear.

The reason for hypertension in $\mathrm{n}-\mathrm{STZ}$ model can be one among the following factors such as hyperglycemia, dyslipidaemia and oxidative stress (free radical generation).

Hyperglycemia and dyslipidaemia may not be the strong factors responsible for elevation of hypertension in this nSTZ model, because the gliclazide treatment did not bring about any change in elevated FBG levels or the lipid dismetabolism, thus the hypertension observed in this model can be irrespective of hyperglycemia and dyslipidaemia. However, the IGT and elevated AI may be the reason, but gliclazide treatment brought some significant change only in the later stages of the treatment period.

The oxidative stress, involving imbalance between free radical generation and radical scavenging effect, causes quenching of nitric oxide by free radicals and preventing its vasodilator effect promoting endothelial dysfunction was reported earlier. ${ }^{2,18,19}$ Gliclazide is a general free radical scavenger, was demonstrated in vitro, and this quality of drug may be important in diabetes as reported earlier. ${ }^{20}$ An in vivo and in vitro antioxidant property of gliclazide was demonstrated with increase in antioxidant parameters such as total plasma antioxidant capacity, superoxide dismutase and thiols, and by fall in 8isoprostanes, a marker of lipid oxidation. ${ }^{13}$ The prevention of hypertension in gliclazide treated rats in the present study could be due to free radical scavenging property of gliclazide. The earlier studies in rats also demonstrated beneficial effects of gliclazide in endothelial dysfunction suggesting that the action must be independent of any modification of insulin secretion. ${ }^{21}$

Clinically gliclazide was found to reduce blood pressure (both systolic and diastolic) in diabetic patients where hypertension was induced using L-arginine and in the same study glibenclamide treatment did not bring about any reduction in blood pressure, thus suggesting that gliclazide increase the bioavailability of nitric oxide. ${ }^{22}$

Diabetic patients were more prone to hypertension than non-diabetics. Thus, the gliclazide treatment can prevent development of hypertension which is related to diabetic complications such as oxidative stress, endothelial dysfunction and other vascular changes in diabetic patients. Since HR did not vary much between control and diabetic group in the present study and the effect of Gliclazide on HR was not seen in the n-STZ administered diabetic model. In the present study the SBP in the gliclazide treated group was less than that of control group during the treatment period; however there was no significant difference when compared to the control group.

CK-MB is one of the isoforms of creatine kinase and its concentration is very high in myocardium. ${ }^{23}$ Hence increase in CK-MB and LDH levels represent damage to heart. Thus decrease in CK-MB and LDH activity by gliclazide shows that it prevents myocardial damage. However, reports of increased LDH and CK-MB activity n-STZ model was hardly seen in literature and the beneficial effect of gliclazide seen in the animal studies could be useful clinically in case of diabetic patients prone to heart diseases such as myocardial infarction.

As insulin resistance has been related to loss of adipose mass in $\mathrm{n}-\mathrm{STZ}$ rat model $^{24}$, the increase in the adipose 
mass after gliclazide treatment would have increased the surface area for glucose uptake thus improving IGT at later stage of treatment period.

Gliclazide treatment in n-STZ model was found to be effective in preventing hypertension, creatine kinase and lactate dehydrogenase activity. Also gliclazide was found to have beneficial effects on the impaired glucose tolerance, dyslipidaemia, adiposity index and total fat pad weight. To improve and prevent the cardiovascular risk factors involved in Type II diabetic patients, gliclazide could be clinically beneficial.

\section{REFERENCES}

1. Chakrabarti R, Rajagopalan R. Diabetes and insulin resistance associated disorders: Disease and the therapy. Curr Sci 2002;83:1533-8.

2. Jennings PE. Vascular benefits of gliclazide beyond glycemic control. Metabolism 2000;49;17-20.

3. Riccio A, Lisato G, Vigili de Kreutzenberg, Marchetto S, Turrin M, Tiengo A, et al. Gliclazide potentiates suppression of hepatic glucose production in non-insulin-dependent diabetic patients. Metabolism 1996;45:1196-202.

4. O'Brien RC, Luo M, Balazs N, Mercuri J. In vitro and in vivo antioxidant properties of gliclazide. J Diabetes Complications 2000;14:201-6.

5. Sliwinska A, Blasiak J, Kasznicki J, Drzewoski J. In vitro effect of gliclazide on DNA damage and repair in patients with type 2 diabetes mellitus (T2DM). Chem Biol Interact 2008;173:159-65.

6. Signorini AM, Fondelli C, Renzoni E, Puccetti C, Gragnoli G, Giorgi G. Antioxidant effects of gliclazide, glibenclamide, and metformin in patients with type 2 diabetes mellitus. Curr Ther Res Clin Exp 2002;63:411-20.

7. Fava D, Cassone-Faldetta M, Laurenti O, De Luca O, Ghiselli A, De Mattia G. Gliclazide improves antioxidant status and nitric oxide-mediated vasodilatation in type 2 diabetes. Diabet Med 2002; 19:752-7.

8. Satyanarayana S, Kumar KE, Sekhar JR. Effect of $\alpha$ lipoic acid on gliclazide-induced hypoglycemia/antihyperglycemia in normal/alloxaninduced diabetic rats. Therapy 2006;3:613-7.

9. Srinivasan K, Viswanad B, Asrat L, Kaul CL, Ramarao P. Combination of High fat-diet fed and low-dose streptozotocin-treated rat: A model for type 2 diabetes and pharmacological screening. Pharmacol Res 2005;52:313-20.

10. Portha B, Serradas P. Improvement in glucoseinduced insulin secretion in diabetic rats after longterm gliclazide treatment: A comparative study using different models of non-insulin dependent diabetes mellitus induced by neonatal Streptozotocin. Am J Med 1991;90(Suppl 6A):15S-21S.
11. Bak JF, Pedersen O. Gliclazide and insulin action in human muscle. Diabetes Res Clin Pract 1991;14 Suppl 2:S61-4.

12. Hoich RI, Frank M. Insulin-potentiating action of gliclazide. Pharmacol Res Commun 1986;18:419-30.

13. Dachicourt N, Baibe D, Gangnerau M, Serradas P, Ravel D, Portha B. Effect of gliclazide on insulin secretion and $\beta$-cell mass in non-insulin dependent diabetic Goto-Kakisaki rats. Eur J Pharmacol 1998;361:243-51.

14. Couturier E. Gliclazide on long-term therapy increases response to glucose of type II diabetics. Diabetes Res Clin Pract 1985;1:343-7.

15. Ohnota H, Kitamura T, Kinukawa M, Hamano S, Shibata N, Miyata H, et al. A rapid and short-acting hypoglycemic agent KAD-1229 improves postprandial hyperglycemia and diabetic complicaions in streptozotocin-induced non-insulin dependent diabetes mellitus rats. Jpn J Pharmacol 1996;71:31523.

16. Tanira MOM, Furman BL. The in vivo interaction between gliclazide and glibenclamide and insulin on glucose disposal in the rat. Pharmacol Res 1999;39:349-56.

17. Marathe PA, Parekar RP, Shinde SP, Rege NN. A split dose regimen of Streptozotocin to induce diabetes in neonatal rat model. Indian $\mathbf{J}$ Pharmacol 2006;38:432-3.

18. Maritim AC, Sanders RA, Watkins III JB. Diabetes, oxidative stress, and antioxidants: A review. J Biochem Mol Toxicol 2003;17:24-38.

19. Schafer A, Alp NJ, Cai S, Lygate CA, Neubauer S, Eigenthaler $M$, et al. Reduced vascular NO bioavailability in diabetes increases platelet activation in vivo. Arterioscler Thromb Vasc Biol 2004;24:1720-6.

20. Scott NA, Jennings PE, Brown J, Belch JJF. Gliclazide: A general free radical scavenger. Eur $\mathbf{J}$ Pharmacol 1991;208:175-7.

21. Vallejo S, Angulo J, Peiro C, Sanchez-Ferrer A, Cercas E, Llergo JL, et al. Prevention of endothelial dysfunction in streptozotocin-induced diabetic rats by gliclazide treatment. J Diabetes Complications 2000;14:224-33.

22. Sikora A, Blasiak J, Drzewoski J. Gliclazide-Oral hypoglycemic drug with pleiotropic mechanism of action. Diabetologia Doświadczalna i Kliniczna 2006;6:1-5.

23. Pal S. Estimation of CKMB from RIQAS control serum at 37 degree celsius. Indian J Clin Biochem 2002; 17:88-90.

24. Takada J, Machado MA, Peres SB, Brito LC, Borges-Silva CN, Costa CEM, et al. Neonatal streptozotocin-induced diabetes mellitus: A model of insulin resistance associated with loss of adipose mass. Metabolism 2007;56:977-84.

doi: 10.5455/2319-2003.ijbcp003612

Cite this article as: Salman IM, Inamdar MN. Effect of gliclazide on cardiovascular risk factors involved in split-dose streptozotocin induced neonatal rat model: a chronic study. Int J Basic Clin Pharmacol 2012;1:196-201. 